\title{
FUNCTIONAL LIMIT THEOREMS FOR CRITICAL PROCESSES WITH IMMIGRATION
}

\author{
I. RAHIMOV, ${ }^{*}$ King Fahd University of Petroleum \& Minerals
}

\begin{abstract}
We consider a critical discrete-time branching process with generation dependent immigration. For the case in which the mean number of immigrating individuals tends to $\infty$ with the generation number, we prove functional limit theorems for centered and normalized processes. The limiting processes are deterministically time-changed Wiener, with three different covariance functions depending on the behavior of the mean and variance of the number of immigrants. As an application, we prove that the conditional least-squares estimator of the offspring mean is asymptotically normal, which demonstrates an alternative case of normality of the estimator for the process with nondegenerate offspring distribution. The norming factor is $n \sqrt{\alpha(n)}$, where $\alpha(n)$ denotes the mean number of immigrating individuals in the $n$th generation.
\end{abstract}

Keywords: Branching process; immigration; functional; martingale limit theorem; Skorokhod space; least-squares estimator 2000 Mathematics Subject Classification: Primary 60J80

Secondary 62F12; 60G99

\section{Introduction}

We consider a discrete-time branching stochastic process $Z(n), n \geq 0, Z(0)=0$. It can be defined by two families of independent, nonnegative integer-valued random variables $\left\{X_{n i}, n, i \geq 1\right\}$ and $\left\{\xi_{k}, k \geq 1\right\}$ recursively as

$$
Z(n)=\sum_{i=1}^{Z(n-1)} X_{n i}+\xi_{n}, \quad n \geq 1 .
$$

Assume that the $X_{n i}$ have a common distribution for all $n$ and $i$, and that the families $\left\{X_{n i}\right\}$ and $\left\{\xi_{n}\right\}$ are independent. The variables $X_{k i}$ will be interpreted as the number of offspring of the $i$ th individual in the $(k-1)$ th generation, and $\xi_{k}$ will denote the number of immigrating individuals in the $k$ th generation. Then $Z(n)$ can be viewed as the size of the $n$th generation of the population. In this interpretation $A=\mathrm{E} X_{n i}$ denotes the mean number of offspring of a single individual. The process $Z(n)$ is called subcritical, critical, or supercritical depending on whether $A<1, A=1$, or $A>1$, respectively. The independence assumption of the families $\left\{X_{n i}\right\}$ and $\left\{\xi_{n}\right\}$ means that reproduction and immigration processes are independent. However, in contrast to the classical models, we do not assume that the $\xi_{n}, n \geq 1$, are identically distributed, i.e. the immigration rate may depend on the time of immigration.

Investigations show that the asymptotic behavior of the process with immigration is very sensitive to any changes of the immigration process in time. For instance, in the critical case, a change in the mean number of immigrating individuals in time leads to such fluctuations of the

Received 8 May 2006; revision received 28 January 2007.

* Postal address: Department of Mathematical Sciences, King Fahd University of Petroleum \& Minerals, Box 1339, Dhahran 31261, Saudi Arabia. Email address: rahimov@kfupm.edu.sa 
process that it is necessary to use various functional normalizations to obtain a nondegenerate limit distribution for the process; see [16, Chapter III] and the references therein. Therefore, a description of the processes that can be used as an approximation in this situation is of interest. Conversely, these kinds of functional limit theorem are useful in estimating parameters and in the study of various functionals of the process.

In this paper we prove functional limit theorems for critical processes for the case in which the immigration mean tends to $\infty$. It turns out that a suitably normalized process may be approximated by a Gaussian process with independent increments and with three different covariance functions depending on the behavior of the mean and variance of the number of immigrants. The limiting Gaussian process can be obtained from the Wiener process by a deterministic time change. As an example of the application of functional limit theorems, we prove that the conditional least-squares estimator (CLSE) of the offspring mean, $A$, is asymptotically normal. It is interesting to note that the norming factor depends on the mean number of immigrants and can be chosen to depend on the rate of immigration.

First approximation theorems of branching stochastic processes have appeared in [5], where it was demonstrated that a branching stochastic process without immigration can be approximated by a diffusion process. Lamperti [12], [13] proved that finite-dimensional distributions of the process with a large number of initial individuals converges to those of some diffusion processes with two different normalizations. These results were extended to the functional form in [14] and [15]. The convergence of finite-dimensional distributions of a sequence of Galton-Watson branching processes with stationary immigration has been investigated in [1] and [11]. Wey and Winnicki [18] have shown that random step functions of a critical branching process with immigration converges in Skorokhod topology to a nonnegative diffusion process. Fluctuation theorems for the sequence of nearly critical branching processes have been proved by Sriram [17], who obtained a diffusion approximation. In [7] and [8] the authors demonstrated that Sriram's result was also valid when the offspring variance tended to 0 , and that the centralized process can be approximated by Ornstein-Uhlenbeck-type processes. In addition, asymptotic normality of the mean-square estimator of the offspring mean was proved with normalizing factor $n^{3 / 2}$. Note that in the latter case the reproduction process will approach a deterministic multiplication of individuals. The same authors also examined the critical branching process with varying offspring and immigration distributions in [9]. However, in contrast to our situation, in [9] the offspring variance tends to 0 .

It was known that in the critical or nearly critical case the CLSE of the offspring mean is not asymptotically normal; see [17] and [18]. When the process is nearly critical and the offspring variance tends to 0 , the results of [7] and [8] have shown that it has a normal limiting distribution. Our Theorem 4 demonstrates an alternative situation of asymptotic normality of the CLSE for the process with nondegenerate offspring distribution.

It is known that (see [2], [4], and [6]) in the case of the Bernoulli offspring distribution the process (1) is an integer-valued, first-order autoregressive (INAR(1)) time-series model with noise $\xi_{k}$. In the framework considered here, the process $Z(n)$ can be related to the $\operatorname{INAR}(1)$ model with nonstationary (rising) noise.

In the proofs we follow the approach introduced by Ispàny et al. [7], [8], and use some tricks of the proof of appropriate statements there. Namely, we represent our process in the form of normalized martingale differences, and use martingale limit theorems to derive our results.

The main results and some examples will be given in Section 2. In Section 3 we prove several preliminary results which will be used in the proofs of the main theorems. Section 4 is devoted to the proofs of the main results. 


\section{Main results and examples}

From now on we assume that $A=\mathrm{E} X_{n i}$ and $B=\operatorname{var}\left(X_{n i}\right)$ are finite. We also assume that $\alpha(n)=\mathrm{E} \xi_{n}<\infty$ and $\beta(n)=\operatorname{var}\left(\xi_{n}\right)<\infty$ for each $n \geq 1$ are regularly varying functions as $n$ tends to $\infty$, i.e. they have the form

$$
\alpha(n)=n^{\alpha} L_{\alpha}(n), \quad \beta(n)=n^{\beta} L_{\beta}(n),
$$

where $\alpha, \beta \geq 0$, and $L_{\alpha}(n)$ and $L_{\beta}(n)$ are slowly varying functions as $n$ tends to $\infty$. Then $A(n)=\mathrm{E} Z(n)$ and $B^{2}(n)=\operatorname{var}(Z(n))$ are finite for each $n \geq 1$ and, when $A=1$,

$$
A(n)=\sum_{k=1}^{n} \alpha(k), \quad B^{2}(n)=\Delta^{2}(n)+\sigma^{2}(n),
$$

where

$$
\Delta^{2}(n)=B \sum_{k=1}^{n} \alpha(k)(n-k), \quad \sigma^{2}(n)=\sum_{k=1}^{n} \beta(k) .
$$

For each $t \in \mathbb{R}_{+}=[0, \infty)$, we define a sequence of step functions

$$
Y_{n}(t)=\frac{Z([n t])-A([n t])}{B(n)} .
$$

Throughout the paper, ' $\stackrel{\mathrm{D}}{\rightarrow}$, ' $\stackrel{\mathrm{d}}{\rightarrow}$ ', and ' $\stackrel{\mathrm{P}}{\rightarrow}$ ' will denote the convergence of random functions in Skorohkod topology, the convergence of random variables in distribution, and convergence in probability, respectively.

Theorem 1. If $A=1, B \in(0, \infty), \alpha(n) \rightarrow \infty$, and $\beta(n)=o(n \alpha(n))$ then $Y_{n}(t) \stackrel{\mathrm{D}}{\rightarrow} W\left(t^{2+\alpha}\right)$ as $n \rightarrow \infty$ weakly in Skorokhod space $D\left(\mathbb{R}_{+}, \mathbb{R}\right)$, where $\left(W(t), t \in \mathbb{R}_{+}\right)$is a standard Brownian motion.

In Theorem 1 note that we do not require a Lindeberg-type condition on the offspring or immigration distribution. In fact, in this case it is satisfied for the immigration process owing to the normalization by $B^{2}(n)$. However, in the opposite case we need one on the sequence $\left\{\xi_{n}, n \geq 1\right\}$, which seems natural for the process with inhomogeneous immigration. Thus, we denote, for each $\varepsilon>0$,

$$
\delta_{n}(\varepsilon)=\frac{1}{\sigma^{2}(n)} \sum_{k=1}^{n} \mathrm{E}\left[\left(\xi_{k}-\alpha(k)\right)^{2} ;\left|\xi_{k}-\alpha(k)\right|>\varepsilon \sigma(n)\right] .
$$

Theorem 2. If $A=1, B \in(0, \infty), \alpha(n) \rightarrow \infty, \alpha(n)=o\left(n^{-1} \beta(n)\right)$, and $\delta_{n}(\varepsilon) \rightarrow 0$ as $n \rightarrow \infty$ for each $\varepsilon>0$ then $Y_{n}(t) \stackrel{\mathrm{D}}{\rightarrow} W\left(t^{1+\beta}\right)$ as $n \rightarrow \infty$ weakly in Skorokhod space $D\left(\mathbb{R}_{+}, \mathbb{R}\right)$.

The following theorem is related to the case in which $n \alpha(n)$ and $\beta(n)$ have the same rate.

Theorem 3. If $A=1, B \in(0, \infty), \alpha(n) \rightarrow \infty, \beta(n) \sim c n \alpha(n), c \in(0, \infty)$, and $\delta_{n}(\varepsilon) \rightarrow 0$ as $n \rightarrow \infty$ for each $\varepsilon>0$ then $Y_{n}(t) \stackrel{\mathrm{D}}{\rightarrow} W\left(t^{1+\beta}\right)=W\left(t^{2+\alpha}\right)$ as $n \rightarrow \infty$ weakly in Skorokhod space $D\left(\mathbb{R}_{+}, \mathbb{R}\right)$. 
Remarks. 1. Using Lemma 1, given in Section 3, we can see that the condition $\beta(n)=$ $o(n \alpha(n))$ is equivalent to $\sigma^{2}(n)=o\left(\Delta^{2}(n)\right)$, the condition $\alpha(n)=o\left(n^{-1} \beta(n)\right)$ is equivalent to $\Delta^{2}(n)=o\left(\sigma^{2}(n)\right)$, and that $\beta(n) \sim c n \alpha(n)$ as $n \rightarrow \infty$, if and only if $\sigma^{2}(n) \sim \theta B^{2}(n)$ with $\theta=d /(d+(1+\beta) B)$, where $d=c(1+\alpha)(2+\alpha)$.

2. Since $\beta(n) / n \alpha(n)$ is regularly varying with exponent $\beta-1-\alpha$ and has a positive finite limit when $\beta(n) \sim \operatorname{cn} \alpha(n)$ as $n \rightarrow \infty$, we conclude that $\beta=\alpha+1$. This explains the equality $W\left(t^{1+\beta}\right)=W\left(t^{2+\alpha}\right)$ in Theorem 3 .

Now we consider some examples of the immigration process which satisfy the conditions of Theorems 1, 2, and 3 .

Example 1. Let $\xi_{k}, k \geq 1$, be Poisson with mean $\lambda(k) \rightarrow \infty, k \rightarrow \infty$, and regularly varying with exponent $\alpha$. Then

$$
\Delta^{2}(n)=B \sum_{k=1}^{n} \lambda(k)(n-k), \quad \sigma^{2}(n)=\sum_{k=1}^{n} \lambda(k),
$$

and, clearly, $\sigma^{2}(n)=o\left(\Delta^{2}(n)\right)$. In this case we obtain the following result from Theorem 1 .

Corollary 1. If $A=1, B \in(0, \infty)$, and $\xi_{k}, k \geq 1$, are Poisson with mean $\lambda(k) \rightarrow \infty$, $k \rightarrow \infty$, and $(\lambda(k))_{k=1}^{\infty}$ is regularly varying with exponent $\alpha$ then $Y_{n}(t) \stackrel{\mathrm{D}}{\rightarrow} W\left(t^{2+\alpha}\right)$ as $n \rightarrow \infty$ weakly in Skorokhod space $D\left(\mathbb{R}_{+}, \mathbb{R}\right)$.

Example 2. Now let $\xi_{k}, k \geq 1$, have positive geometric distributions with parameter $p_{k}=k^{-1}$, i.e. $\mathrm{P}\left\{\xi_{k}=i\right\}=q_{k}^{i-1} p_{k}, i=1,2, \ldots, q_{k}=1-p_{k}$. In this case

$$
\alpha(k)=k \quad \text { and } \quad \beta(k)=q_{k} p_{k}^{-2}=k^{2}\left(1-k^{-1}\right) .
$$

Consequently, we have $\Delta^{2}(n) \sim B n^{3} / 6$ and $\sigma^{2}(n) \sim n^{3} / 3$. Therefore,

$$
\sigma^{2}(n) \sim \frac{2 B^{2}(n)}{B+2} .
$$

Now we show fulfilment of the Lindeberg condition. Since E $S^{\xi_{k}}=\left(p_{k} S\right)\left(1-q_{k} S\right)^{-1}$, $0<S<1$, we find that

$$
\left(\mathrm{E} S^{\xi_{k}}\right)^{\prime \prime \prime}=6 p_{k} q_{k}^{2}\left(1-q_{k} S\right)^{-4}
$$

where '"', denotes the third derivative. Therefore,

$$
\mathrm{E} \xi_{k}\left(\xi_{k}-1\right)\left(\xi_{k}-2\right)=6 q_{k}^{2} p_{k}^{-3} .
$$

From this we conclude that $\mathrm{E}\left|\xi_{k}-\alpha(k)\right|^{3}=O\left(k^{3}\right), k \rightarrow \infty$, which leads to the relation

$$
C_{n}^{3}=: \sum_{k=1}^{n} \mathrm{E}\left|\xi_{k}-\alpha(k)\right|^{3}=O\left(n^{4}\right), \quad n \rightarrow \infty .
$$

Thus, $C_{n}^{3} / \sigma^{3}(n)=O\left(n^{-1 / 2}\right), n \rightarrow \infty$, i.e. the Lyapunov condition is satisfied for $\xi_{k}, k \geq 1$. Now we obtain the following result from Theorem 3 .

Corollary 2. If $A=1, B \in(0, \infty)$, and $\xi_{k}, k \geq 1$, are geometric with parameter $p_{k}=k^{-1}$ then $Y_{n}(t) \stackrel{\mathrm{D}}{\rightarrow} W\left(t^{3}\right)$ as $n \rightarrow \infty$ weakly in Skorokhod space $D\left(\mathbb{R}_{+}, \mathbb{R}\right)$. 
Example 3. Let $\xi_{k}, k \geq 1$, be such that $p=\mathrm{P}\left\{\xi_{k}=k^{2}\right\}=1-\mathrm{P}\left\{\xi_{k}=0\right\}$ and $q=1-p$. Then simple calculations give

$$
\Delta^{2}(n) \sim \frac{B p n^{4}}{12} \quad \text { and } \quad \sigma^{2}(n) \sim \frac{p q n^{5}}{5} \quad \text { as } n \rightarrow \infty,
$$

and, consequently, $\Delta^{2}(n)=o\left(\sigma^{2}(n)\right)$. Since in this case

$$
C_{n}^{3} \sim \frac{p q\left(p^{2}+q^{2}\right) n^{7}}{7} \text { and } \sigma^{3}(n) \sim\left(\frac{p q}{5}\right)^{3 / 2} n^{15 / 2}
$$

the Lyapunov condition is again satisfied. Thus, from Theorem 2 we find that $Y_{n}(t)$ converges, as $n$ tends to $\infty$, to $W\left(t^{5}\right)$ weakly in Skorokhod space $D(\mathbb{R}+, \mathbb{R})$.

Now we consider one nontrivial application of our theorems, related to the conditional leastsquares estimator of the offspring mean. Let $\mathfrak{I}(n)$ for each $n \geq 0$ be a $\sigma$-algebra generated by $\{Z(k), k=0,1, \ldots, n\}$. From (1) we obtain

$$
\mathrm{E}[Z(n) \mid \mathfrak{I}(n-1)]=A Z(n-1)+\alpha(n), \quad n \geq 1 .
$$

If we assume that the immigration mean, $\alpha(n)$, is known then the CLSE $\hat{A_{n}}$ of $A$ must minimize the sum of squares error

$$
\sum_{k=1}^{n}(Z(k)-A Z(k-1)-\alpha(k))^{2}
$$

By standard arguments we find that it has the form

$$
\hat{A_{n}}=\frac{\sum_{k=1}^{n}(Z(k)-\alpha(k)) Z(k-1)}{\sum_{k=1}^{n} Z^{2}(k-1)} .
$$

Using Theorem 1, we shall prove the following result for $\hat{A_{n}}$.

Theorem 4. If the conditions of Theorem 1 are fulfilled then

$$
n \sqrt{\alpha(n)}\left(\hat{A_{n}}-1\right) \stackrel{\mathrm{d}}{\rightarrow} N(0, a),
$$

where $N(0, a)$ is a normal random variable with mean 0 and variance

$$
a^{2}=\frac{(1+\alpha)(2 \alpha+3)^{2} B}{3 \alpha+4} .
$$

We note that, if $\alpha=0$, i.e. the immigration mean tends to $\infty$ as a slowly varying function, then the variance of the limiting distribution is $9 B / 4$.

In further publications it will be shown that it is possible to study the CLSE in the case when the conditions of Theorems 2 or 3 are satisfied. Note that in this case $\hat{A_{n}}$ may not necessarily be asymptotically normal. 


\section{Preliminary results}

We start with two simple lemmas on regularly varying functions. If a sequence $\left(C_{n}\right)_{n=1}^{\infty}$ or function $f$ is regularly varying with exponent $\rho$, we write $\left(C_{n}\right)_{n=1}^{\infty} \in R_{\rho}$ and $f \in R_{\rho}$. The following result is a discrete form of Karamata's well-known theorem on regularly varying functions; see [3, Theorem 1.5.11].

Lemma 1. If $\left(C_{n}\right)_{n=1}^{\infty} \in R_{\rho}$ then, for any $\theta \in(-\rho-1, \infty)$,

$$
\sum_{k=1}^{n} k^{\theta} C_{k} \sim \frac{n^{\theta+1} C_{n}}{\theta+\rho+1} \quad \text { as } n \rightarrow \infty
$$

and $\left(\sum_{k=1}^{n} k^{\theta} C_{k}\right)_{n=1}^{\infty} \in R_{\theta+\rho+1}$.

Proof. We consider the function $f_{c}:[0, \infty) \mapsto \mathbb{R}$ defined as $f_{c}(x):=C_{[x]}$ for each $x \in$ $[0, \infty)$. Here $[x]$ denotes the integer part of $x$. Owing to Theorem 1.9 .5 of [3], $f_{c} \in R_{\rho}$ and is locally bounded on $[0, \infty)$. Applying Theorem 1.5 .11 of [3], we have

$$
\int_{0}^{x} t^{\theta} f_{c}(t) \mathrm{d} t \sim \frac{x^{\theta+1} f_{c}(x)}{\theta+\rho+1} \quad \text { as } x \rightarrow \infty,
$$

which implies that the function $x \mapsto \int_{0}^{x} t^{\theta} f_{c}(t) \mathrm{d} t$ is regularly varying with exponent $\theta+\rho+1$. Since $C_{n-1} / C_{n} \rightarrow \infty$, we obtain the statements of Lemma 1 .

Lemma 2. If $A(n)$ is a regularly varying function with exponent $\alpha \geq 0$ then

$$
\sup _{\varepsilon \leq t \leq a}\left|\frac{A(n t)}{A(n)}-t^{\alpha}\right| \rightarrow 0 \quad \text { as } n \rightarrow \infty
$$

for any $0<\varepsilon \leq a<\infty$.

The assertion of Lemma 2 is a simple consequence of the uniform convergence theorem for slowly varying functions.

Lemma 3. If $\alpha(n) \rightarrow \infty$ as $n \rightarrow \infty$ and regularly varies with exponent $\alpha$ and $A(n)=$ $\alpha(1)+\cdots+\alpha(n)$ then, as $n \rightarrow \infty$,

(a)

$$
\Delta^{2}(n) \sim \frac{B \alpha(n) n^{2}}{(\alpha+1)(\alpha+2)}, \quad \sigma^{2}(n) \sim \frac{n \beta(n)}{\beta+1}
$$

(b) for each $\gamma \geq 0$,

$$
\sum_{k=1}^{n} A^{\gamma}(k) \sim \frac{n}{\gamma \alpha+\gamma+1} A^{\gamma}(n)
$$

Proof. To prove part (a), consider

$$
\sum_{k=1}^{n} \alpha(k)(n-k)=n A(n)-\sum_{k=1}^{n} k \alpha(k) .
$$

If we apply Lemma 1 with $\theta=0$ and $\theta=1$, we obtain

$$
A(n) \sim \frac{n \alpha(n)}{\alpha+1}, \quad \sum_{k=1}^{n} k \alpha(k) \sim \frac{n^{2} \alpha(n)}{\alpha+2}, \quad \text { as } n \rightarrow \infty,
$$


which implies the first relation in (4). The second relation in (4) is a direct consequence of Lemma 1.

To prove part (b), we take into account the facts that $(A(n))_{n=1}^{\infty} \in R_{\alpha+1}$ and $\left((A(n))^{\gamma}\right)_{n=1}^{\infty} \in$ $R_{(\alpha+1) \gamma}$ for any $\gamma \in[0, \infty)$. Therefore, again applying Lemma 1 with $\theta=0$, we obtain

$$
\sum_{k=1}^{n}(A(k))^{\gamma} \sim \frac{n}{(\alpha+1) \gamma+1}(A(n))^{\gamma} \quad \text { as } n \rightarrow \infty .
$$

This completes the proof.

Lemma 4. If $\alpha(n) \rightarrow \infty$ as $n \rightarrow \infty$ then, for each $t \in \mathbb{R}_{+}=[0, \infty)$,

(a)

$$
B^{-4}(n) \operatorname{var}\left(\sum_{k=1}^{[n t]} Z(k)\right) \rightarrow 0
$$

(b)

$$
B^{-4}(n) \sum_{k=1}^{[n t]} E Z^{2}(k) \rightarrow 0 .
$$

Proof. To prove part (a), we consider

$$
\operatorname{var}\left(\sum_{k=1}^{[n t]} Z(k)\right)=I_{1}+I_{2}
$$

where

$$
I_{1}=\sum_{k=1}^{[n t]} B^{2}(k), \quad I_{2}=2 \sum_{i=1}^{[n t]-1} \sum_{j=i+1}^{[n t]} \operatorname{cov}(Z(i), Z(j)) .
$$

It is easy to see that

$$
B^{-4}(n) I_{1} \leq \frac{B^{2}([n t])[n t]}{B^{4}(n)} .
$$

Owing to Lemma $1, B^{2}(n)$ is regularly varying and

$$
B^{2}(n) \sim \frac{B \alpha(n) n^{2}}{(\alpha+1)(\alpha+2)}+\frac{n \beta(n)}{\beta+1} \quad \text { as } n \rightarrow \infty,
$$

and we find that $I_{1} / B^{4}(n) \rightarrow 0$ as $n \rightarrow \infty$. Since

$$
\operatorname{cov}(Z(t), Z(t+n))=A^{n} \operatorname{var}(Z(t)),
$$

recalling that $B^{2}(n)=\operatorname{var}(Z(n))$, we have

$$
I_{2}=\sum_{k=1}^{[n t]-1}([n t]-i) B^{2}(i) \leq B^{2}([n t])([n t]-1)^{2} .
$$

Again taking (6) into account and the fact that $\alpha(n) \rightarrow \infty$, we conclude that $I_{2} / B^{-4}(n) \rightarrow 0$ as $n \rightarrow \infty$. 
To prove part (b), we observe that

$$
\sum_{k=1}^{[n t]} \mathrm{E} Z^{2}(k)=\sum_{k=1}^{[n t]} \operatorname{var}(Z(k))+\sum_{k=1}^{[n t]} A^{2}(k) .
$$

As it was just proved, the first sum is $o\left(B^{4}(n)\right)$ as $n$ tends to $\infty$. It follows, from Lemma 1 and part (b) of Lemma 3, that

$$
\sum_{k=1}^{n} A^{2}(k) \sim \frac{n}{2 \alpha+3} A^{2}(n), \quad A(n) \sim \frac{n \alpha(n)}{\alpha+1}, \quad \text { as } n \rightarrow \infty .
$$

From (6) and (8), we find that the second sum in (7) is also $o\left(B^{4}(n)\right)$ as $n$ tends to $\infty$. This completes the proof.

Lemma 5. Let $X_{k i}$ be the random variables given in (1), let $\bar{X}_{k i}=X_{k i}-1$, and let $T(k)=$ $\sum_{i=1}^{Z(k-1)} \bar{X}_{k i}$. Then

(a)

$$
\mathrm{E}\left[(T(k))^{2} \mid \Im(k-1)\right]=B Z(k-1)
$$

(b)

$$
\mathrm{E}\left[\left(\Sigma^{\prime} \bar{X}_{k i} \bar{X}_{k j}\right)^{2} \mid \Im(k-1)\right]=2 B^{2} Z(k-1)(Z(k-1)-1),
$$

where $\sum^{\prime}$ denotes summation for $i, j=1,2, \ldots, Z(k-1)$ such that $i \neq j$.

Proof. Equations (9) and (10) are a direct consequence of the independence of the random variables $\bar{X}_{k i}$ and $\bar{X}_{k j}, i \neq j$, and of a simple property of the conditional expectation.

The following technical result is vital in the proofs of the main theorems.

Lemma 6. For any $\theta>0$,

$$
\mathrm{E}\left[(T(k))^{2} \mathbf{1}_{\{|T(k)|>\theta\}} \mid \Im(k-1)\right] \leq I_{1}+I_{2},
$$

where

$$
\begin{gathered}
I_{1}=Z(k-1) \mathrm{E}\left[\bar{X}_{11}^{2} \mathbf{1}_{\left\{\left|\bar{X}_{11}\right|>\theta / 2\right\}}\right], \\
I_{2}=\frac{4 B^{2}}{\theta^{2}}(Z(k-1))^{2}+\frac{2^{1 / 2} B^{3 / 2}}{\theta}(Z(k-1))^{3 / 2} .
\end{gathered}
$$

Proof. If we use the simple inequality

$$
\mathbf{1}_{\{|\xi+\eta|>\varepsilon\}} \leq \mathbf{1}_{\{|\xi|>\varepsilon / 2\}}+\mathbf{1}_{\{|\eta|>\varepsilon / 2\}}
$$

the left-hand side of (11) can be estimated by $R_{1}+R_{2}+R_{3}$, with

$$
\begin{aligned}
& R_{1}=\mathrm{E}\left[\sum_{i=1}^{Z(k-1)} \bar{X}_{k i}^{2} \mathbf{1}_{\left\{\left|\bar{X}_{k i}\right|>\theta / 2\right\}} \mid \Im(k-1)\right]=Z(k-1) \mathrm{E}\left[\bar{X}_{11}^{2} \mathbf{1}_{\left\{\left|\bar{X}_{11}\right|>\theta / 2\right\}}\right], \\
& R_{2}=\mathrm{E}\left[\sum_{i=1}^{Z(k-1)} \bar{X}_{k i}^{2} \mathbf{1}_{\left\{\left|\tau_{k i}\right|>\theta / 2\right\}} \mid \Im(k-1)\right], \\
& R_{3}=\mathrm{E}\left[\Sigma^{\prime} \bar{X}_{k i} \bar{X}_{k j} \mathbf{1}_{\{|T(k)|>\theta\}} \mid \mathfrak{I}(k-1)\right],
\end{aligned}
$$


where $\Sigma^{\prime}$ is as defined in Lemma 5 , and $\tau_{k i}=T(k)-\bar{X}_{k i}$. To estimate $R_{2}$ we use the independence of $\bar{X}_{k i}$ and $\tau_{k i}$, the Chebyshev inequality, and (9), and we obtain

$$
R_{2} \leq \frac{4 B}{\theta^{2}} \sum_{i=1}^{Z(k-1)} \mathrm{E}\left[\tau_{k i}^{2} \mid \Im(k-1)\right]=\frac{4 B^{2}}{\theta^{2}} Z(k-1)(Z(k-1)-1) .
$$

Using the Cauchy-Schwarz and Chebyshev inequalities, (9), and (10), we see that $R_{3}$ is dominated by

$$
\begin{gathered}
\frac{1}{\theta}\left(\mathrm{E}\left[\left(\Sigma^{\prime} \bar{X}_{k i} \bar{X}_{k j}\right)^{2} \mid \Im(k-1)\right] \mathrm{E}\left[(T(k))^{2} \mid \Im(k-1)\right]\right)^{1 / 2} \\
=\frac{2^{1 / 2} B^{3 / 2} Z(k-1)}{\theta} \sqrt{Z(k-1)-1} .
\end{gathered}
$$

This completes the proof.

\section{Proofs of Theorems 1-4}

We represent our process $Y_{n}(t)$ in the form of a sum of normalized martingale differences, and deduce our results from a martingale limit theorem. Let $M(k)=Z(k)-\mathrm{E}[Z(k) \mid \mathfrak{I}(k-1)]$. Then it follows, from (1) and (2), that

$$
Z(k)-\mathrm{E}[Z(k)]=Z(k-1)-\mathrm{E}[Z(k-1)]+M(k) .
$$

Subsequent application of this identity leads to the relation

$$
Y_{n}(t)=\sum_{k=1}^{[n t]} \frac{M(k)}{B(n)} .
$$

To prove Theorem 1, we use the following version of the martingale central limit theorem from [10].

Theorem 5. ([10, Theorem VIII].) Let $\left\{U_{k}^{n}, k \geq 1\right\}$ for each $n \geq 1$ be a sequence of martingale differences with respect to some filtration $\left\{\mathfrak{I}_{k}^{n}, k \geq 1\right\}$, such that the conditional Lindeberg condition

$$
\sum_{k=1}^{[n t]} \mathrm{E}\left[\left(U_{k}^{n}\right)^{2} \mathbf{1}_{\left\{\left|U_{k}^{n}\right|>\varepsilon\right\}} \mid \mathfrak{I}_{k-1}^{n}\right] \stackrel{\mathrm{P}}{\rightarrow} 0
$$

holds as $n \rightarrow \infty$ for all $\varepsilon>0$ and $t \in \mathbb{R}_{+}$. Then

$$
\sum_{k=1}^{[n t]} U_{k}^{n} \stackrel{\mathrm{D}}{\rightarrow} U(t)
$$

as $n \rightarrow \infty$ weakly, where $U(t)$ is a continuous Gaussian martingale with mean 0 and covariance function $C(t), t \in \mathbb{R}_{+}$, if and only if

$$
\sum_{k=1}^{[n t]} \mathrm{E}\left[\left(U_{k}^{n}\right)^{2} \mid \mathfrak{I}_{k-1}^{n}\right] \stackrel{\mathrm{P}}{\rightarrow} C(t) \quad \text { as } n \rightarrow \infty
$$

for each $t \in \mathbb{R}_{+}$. 
Proof of Theorem 1. First we prove fulfilment of condition (14) for the sum in (13) with $U_{k}^{n}=M(k) / B(n)$ and $\mathfrak{I}_{k}^{n}=\mathfrak{I}(k)$ for all $n \geq 1$. Since

$$
M(k)=\sum_{i=1}^{Z(k-1)}\left(X_{k i}-1\right)+\xi_{k}-\alpha(k)
$$

we easily obtain

$$
\sum_{k=1}^{[n t]} \mathrm{E}\left[\left(U_{k}^{n}\right)^{2} \mid \Im_{k-1}^{n}\right]=\frac{\sigma^{2}([n t])}{B^{2}(n)}+\frac{B}{B^{2}(n)} \sum_{k=1}^{[n t]} Z(k-1) .
$$

From Lemma 4 it follows that the variance of the second sum in (16) tends to 0 as $n$ tends to $\infty$. Therefore, we just consider

$$
\frac{B}{B^{2}(n)} \sum_{k=1}^{[n t]} \mathrm{E}[Z(k-1)]=\frac{B}{B^{2}(n)} \sum_{k=1}^{[n t]} A(k-1) .
$$

Since $B^{2}(n) \sim \Delta^{2}(n)$ as $n \rightarrow \infty$ under the conditions of Theorem 1, appealing to Lemma 3, we see that the last sum converges in probability to $t^{2+\alpha}$ as $n$ tends to $\infty$. Hence, condition (14) is satisfied with $C(t)=t^{2+\alpha}$.

In our case the Lindeberg condition will be satisfied if, for each $\varepsilon>0$,

$$
I(n)=\frac{1}{B^{2}(n)} \sum_{k=1}^{[n t]} \mathrm{E}\left[(M(k))^{2} \mathbf{1}_{\{|M(k)|>\varepsilon B(n)\}} \mid \mathfrak{I}(k-1)\right] \stackrel{\mathrm{P}}{\rightarrow} 0 \quad \text { as } n \rightarrow \infty .
$$

Taking (15) into account and the independence of the immigration and reproduction processes, we have

$$
I(n)=I_{1}(n)+I_{2}(n),
$$

where

$$
\begin{aligned}
& I_{1}(n)=\frac{1}{B^{2}(n)} \sum_{k=1}^{[n t]} \mathrm{E}\left[(T(k))^{2} \mathbf{1}_{\{|M(k)|>\varepsilon B(n)\}} \mid \Im(k-1)\right], \\
& I_{2}(n)=\frac{1}{B^{2}(n)} \sum_{k=1}^{[n t]} \mathrm{E}\left[\left(\xi_{k}-\alpha(k)\right)^{2} \mathbf{1}_{\{|M(k)|>\varepsilon B(n)\}} \mid \mathfrak{I}(k-1)\right],
\end{aligned}
$$

and $T(k)$ is as defined in Lemma 5.

Consider $I_{1}(n)$. Using inequality (12), we see that it can be estimated by the sum $I_{11}(n)+$ $I_{12}(n)$, where

$$
\begin{aligned}
& I_{11}(n)=\frac{1}{B^{2}(n)} \sum_{k=1}^{[n t]} \mathrm{E}\left[(T(k))^{2} \mathbf{1}_{\{|T(k)|>\varepsilon B(n) / 2\}} \mid \mathfrak{I}(k-1)\right],
\end{aligned}
$$

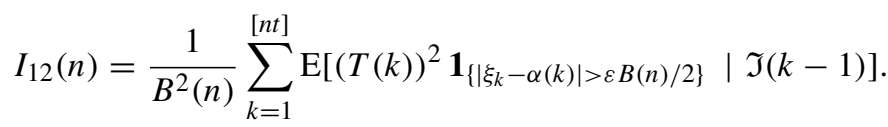


Owing to Lemma 6, we obtain

$I_{11}(n) \leq \frac{\Upsilon(\varepsilon B(n) / 4)}{B^{2}(n)} \sum_{k=1}^{[n t]} Z(k-1)+\frac{16 B^{2}}{\varepsilon^{2} B^{4}(n)} \sum_{k=1}^{[n t]}(Z(k-1))^{2}+\frac{2 B}{\varepsilon B^{3}(n)} \sum_{k=1}^{[n t]}(Z(k-1))^{3 / 2}$,

where $\Upsilon_{n}(\varepsilon)=\mathrm{E}\left[\bar{X}_{11}^{2} \mathbf{1}_{\left\{\left|\bar{X}_{11}\right|>\varepsilon\right\}}\right]$. As it was proved, the sum in the first term divided by $B^{2}(n)$ converges in probability to $t^{2+\alpha}$ as $n$ tends to $\infty$. Owing to (5), the second term converges in probability to 0 as $n$ tends to $\infty$. To estimate the last term we use the inequality

$$
\sum_{k=1}^{n} a_{k} b_{k} \leq \sqrt{\sum_{k=1}^{n} a_{k}^{2} \sum_{k=1}^{n} b_{k}^{2}}
$$

We find that this inequality is not greater than

$$
\frac{2 B}{\varepsilon} \sqrt{\frac{1}{B^{4}(n)} \sum_{k=1}^{[n t]}(Z(k-1))^{2}} \sqrt{\frac{1}{B^{2}(n)} \sum_{k=1}^{[n t]} Z(k-1)}
$$

and, therefore, also converges in probability to 0 as $n$ tends to $\infty$. Hence, we have proved that $I_{11}(n) \stackrel{\mathrm{P}}{\rightarrow} 0$ as $n \rightarrow \infty$ for any $\varepsilon>0$.

Consider $I_{12}(n)$. Using the independence of the immigration and reproduction processes, the Chebyshev inequality, and (9), we have

$$
I_{12}(n) \leq \frac{4 B}{\varepsilon^{2} B^{4}(n)} \sum_{k=1}^{[n t]} Z(k-1) \beta(k) .
$$

Taking into account the inequality

$$
\sum_{k=1}^{[n t]} \mathrm{E}[Z(k-1)] \beta(k) \leq A([n t]) \sigma^{2}([n t]),
$$

we find that $I_{12}(n) \stackrel{\text { P }}{\rightarrow} 0$ as $n \rightarrow \infty$.

To estimate $I_{2}(n)$, we take into account the fact that $I_{2}(n) \leq \sigma^{2}([n t]) / B^{2}(n)$ and, owing to the condition $\sigma^{2}(n)=o\left(\Delta^{2}(n)\right)$, we conclude that $I_{2}(n) \stackrel{\mathrm{P}}{\rightarrow} 0$ as $n \rightarrow \infty$. This completes the proof.

Proof of Theorem 2. We again prove fulfilment of the conditions of Theorem 5. Since $B^{2}(n) \sim \sigma^{2}(n)$ as $n \rightarrow \infty$ under the conditions of Theorem 2, the first term in (16) tends to $t^{1+\beta}$ as $n$ tends to $\infty$. Owing to Lemma 3,

$$
\sum_{k=1}^{[n t]} \mathrm{E}[Z(k-1)] \sim \frac{\text { constant }}{n} \Delta^{2}(n) \quad \text { as } n \rightarrow \infty,
$$

and appealing to Lemma 4, we find that the second term in (16) converges in probability to 0 as $n$ tends to $\infty$. Hence, condition (14) is satisfied with $C(t)=t^{1+\beta}$. 
In the proof of the fulfilment of the Lindeberg condition, convergence in probability to 0 of the term $I_{1}(n)$ in (17) will remain true under the conditions of Theorem 2. Consider $I_{2}(n)$. Using inequality (12), we find that

$$
I_{2}(n) \leq \delta_{n}\left(\frac{\varepsilon}{2}\right)+\frac{1}{B^{2}(n)} \sum_{k=1}^{[n t]} \beta(k) \mathrm{P}\left\{|T(k)|>\frac{\varepsilon B(n)}{2} \mid \mathfrak{I}(k-1)\right\} .
$$

It follows, from the Chebyshev inequality and (9), that the second term in (20) is dominated by

$$
\frac{4 B}{\varepsilon^{2} B^{4}(n)} \sum_{k=1}^{n} \beta(k) Z(k-1) .
$$

Using (18), we can prove that the second term converges in probability to 0 as $n$ tends to $\infty$. Consequently, $I_{2}(n) \stackrel{\text { P }}{\rightarrow} 0$ as $n \rightarrow \infty$ for each $\varepsilon>0$ under the conditions of Theorem 2 . This completes the proof.

Proof of Theorem 3. Under the conditions of Theorem 3, the first term in (16) tends to $\theta t^{1+\beta}$. Using (19), Lemma 4, and taking Remark 1 into account, we find that the second term converges in probability to $(1-\theta) t^{2+\alpha}$ as $n$ tends to $\infty$. Consequently, condition (14) is satisfied with $C(t)=\theta t^{1+\beta}+(1-\theta) t^{2+\alpha}=t^{2+\alpha}$.

The fulfilment of the Lindeberg condition can be proved in a similar manner to that given in the proofs of the previous theorems. Therefore, its proof is omitted. The assertion of Theorem 3 again follows from Theorem 5.

Proof of Theorem 4. From (3) we obtain

$$
\hat{A}_{n}-1=\frac{\sum_{k=1}^{n} Z(k-1) M(k)}{\sum_{k=1}^{n} Z^{2}(k-1)}=: \frac{D(n)}{Q(n)} .
$$

Rewrite $D(n)$ as $D(n)=D_{1}(n)+D_{2}(n)$, where

$$
D_{1}(n)=\sum_{k=2}^{n} \sum_{i=1}^{k-1} M(i) M(k) \quad \text { and } \quad D_{2}(n)=\sum_{k=2}^{n} A(k-1) M(k) .
$$

Consider $D_{1}(n)$. Since

$$
D_{1}(n)=\sum_{k=1}^{n} \sum_{i=1}^{k} M(i) M(k)-\sum_{k=1}^{n} M^{2}(k),
$$

using the simple identity

$$
\left(\sum_{k=1}^{n} M(k)\right)^{2}=D_{1}(n)+\sum_{k=1}^{n} \sum_{i=1}^{k} M(i) M(k),
$$

we obtain

$$
D_{1}(n)=\frac{B^{2}(n)}{2} Y_{n}^{2}(1)-\frac{1}{2} \sum_{k=1}^{n} M^{2}(k)
$$


It was shown, in the proof of Theorem 1, that

$$
\frac{1}{B^{2}(n)} \sum_{k=1}^{[n t]} \mathrm{E}\left[M^{2}(k) \mid \Im(k-1)\right] \stackrel{\mathrm{P}}{\rightarrow} t^{2+\alpha} \quad \text { as } n \rightarrow \infty
$$

for each $t \in R_{+}$. Using this and the facts that $B^{2}(n) \sim \Delta^{2}(n)$ and $A(n) \sim C \Delta(n) \sqrt{\alpha(n)}$ as $n \rightarrow \infty$, where $C$ is a positive constant, we can prove that

$$
\frac{1}{K(n)} \sum_{k=1}^{n} M^{2}(k) \stackrel{\mathrm{P}}{\rightarrow} 0 \quad \text { as } n \rightarrow \infty
$$

where $K(n)=A(n) \Delta(n)$. It follows, from Theorem 1, that $Y_{n}^{2}(1) \stackrel{\mathrm{D}}{\rightarrow} W^{2}(1)$ as $n \rightarrow \infty$. Taking this into account in (22), we find that, as $n \rightarrow \infty$,

$$
\frac{1}{K(n)} D_{1}(n) \stackrel{\mathrm{P}}{\rightarrow} 0 .
$$

Now we consider $D_{2}(n)$. It is not difficult to see that

$$
D_{2}(n)=\sum_{k=2}^{n} \sum_{i=1}^{k-1} \alpha(i) M(k)=\sum_{i=1}^{n-1} \alpha(i) \sum_{k=i+1}^{n} M(k) .
$$

Therefore, taking (13) into account, it can be written as

$$
\frac{1}{K(n)} D_{2}(n)=\int_{0}^{1}\left(Y_{n}(1)-Y_{n}(t)\right) \mathrm{d} A_{n}(t),
$$

where $A_{n}(t)=A([n t]) / A(n), n \geq 1$, are nondecreasing functions of $t$.

Now we consider the sequence of functionals $\Psi_{n}: D\left(\mathbb{R}_{+}, \mathbb{R}\right) \mapsto \mathbb{R}, n \geq 1$, defined by

$$
\Psi_{n}(x)=\int_{0}^{1}(x(1)-x(t)) \mathrm{d} A_{n}(t) .
$$

Since $A_{n}(t) \rightarrow t^{1+\alpha}$ as $n \rightarrow \infty$ uniformly on compact subsets of $[0, \infty)$, for all $x, x_{n} \in D\left(\mathbb{R}_{+}, \mathbb{R}\right)$ such that $\sup \left|x_{n}-x\right| \rightarrow 0, n \rightarrow \infty$, we have $\left|\Psi_{n}\left(x_{n}\right)-\Psi(x)\right| \rightarrow 0$ as $n \rightarrow \infty$, where

$$
\Psi(x)=(1+\alpha) \int_{0}^{1}(x(1)-x(t)) t^{\alpha} \mathrm{d} t .
$$

It follows, from Theorem 1 and Lemma 4.1 of [7], that $\Psi_{n}\left(Y_{n}\right) \stackrel{\mathrm{D}}{\rightarrow} \Psi\left(W\left(t^{2+\alpha}\right)\right)$ as $n \rightarrow \infty$. Hence, we conclude that

$$
\frac{1}{K(n)} D_{2}(n) \stackrel{\mathrm{D}}{\rightarrow} \eta \quad \text { as } n \rightarrow \infty
$$

where

$$
\eta=W(1)-(1+\alpha) \int_{0}^{1} W\left(t^{2+\alpha}\right) t^{\alpha} \mathrm{d} t .
$$


Now we consider $Q(n)$. It can be written as $Q(n)=Q_{1}(n)+2 Q_{2}(n)+Q_{3}(n)$, where

$$
\begin{gathered}
Q_{1}(n)=\sum_{k=1}^{n} A^{2}(k-1), \\
Q_{2}(n)=\sum_{k=1}^{n} A(k-1)(Z(k-1)-A(k-1)), \\
Q_{3}(n)=\sum_{k=1}^{n}(Z(k-1)-A(k-1))^{2} .
\end{gathered}
$$

It follows, from Lemma 3, that

$$
\lim _{n \rightarrow \infty} \frac{Q_{1}(n)}{n A^{2}(n)}=(2 \alpha+3)^{-1} .
$$

To estimate $Q_{2}(n)$, we consider

$$
\frac{Q_{2}(n)}{n K(n)}=\sum_{k=1}^{n} A_{n}\left(\frac{k}{n}\right) \int_{k / n}^{(k+1) / n} Y_{n}(t) \mathrm{d} t
$$

Now we define the functionals $\Phi_{n}: D\left(\mathbb{R}_{+}, \mathbb{R}\right) \mapsto \mathbb{R}, n \geq 1$, by

$$
\Phi_{n}(x)=\sum_{k=1}^{n-1} A_{n}\left(\frac{k}{n}\right) \int_{k / n}^{(k+1) / n} x(t) \mathrm{d} t .
$$

It is easy to see that, for any $x, x_{n} \in D\left(\mathbb{R}_{+}, \mathbb{R}\right)$ such that $\sup \left|x_{n}-x\right| \rightarrow 0, n \rightarrow \infty$, we have $\left|\Phi_{n}\left(x_{n}\right)-\Phi(x)\right| \rightarrow 0$ as $n \rightarrow \infty$, where

$$
\Phi(x)=\int_{0}^{1} t^{1+\alpha} x(t) \mathrm{d} t .
$$

We have

$$
\frac{Q_{2}(n)}{n K(n)}=\int_{0}^{1} A_{n}(t) Y_{n}(t) \mathrm{d} t=\Psi_{n}\left(Y_{n}\right),
$$

where $\Psi_{n}(x):=\int_{0}^{1} A_{n}(t) x(t) \mathrm{d} t$. Therefore, again using Theorem 1 and Lemma 4.1 of [7], we conclude that

$$
\frac{Q_{2}(n)}{n K(n)} \stackrel{\mathrm{D}}{\rightarrow} \int_{0}^{1} t^{1+\alpha} W\left(t^{2+\alpha}\right) \mathrm{d} t .
$$

Since $A^{2}(n) / K(n) \sim \sqrt{(2+\alpha) \alpha(n)} / \sqrt{B(1+\alpha)}$ as $n \rightarrow \infty$, we have

$$
\frac{Q_{2}(n)}{n A^{2}(n)} \stackrel{\mathrm{P}}{\rightarrow} 0 \quad \text { as } n \rightarrow \infty .
$$

Now we consider

$$
\frac{Q_{3}(n)}{n B^{2}(n)}=\int_{0}^{1} Y_{n}^{2}(t) \mathrm{d} t
$$


It follows, from Theorem 1 and the continuous mapping theorem, that the right-hand side of the last equality converges in distribution to $\int_{0}^{1} W^{2}\left(t^{2+\alpha}\right) \mathrm{d} t$ as $n$ tends to $\infty$. Therefore, taking (6) into account and the second relation in (8), we conclude that

$$
\frac{Q_{3}(n)}{n A^{2}(n)} \stackrel{\mathrm{P}}{\rightarrow} 0 \quad \text { as } n \rightarrow \infty
$$

Now from (21), (23), (24), and (25)-(28), we find that, as $n \rightarrow \infty$,

$$
n \sqrt{\alpha(n)}\left(\hat{A}_{n}-1\right) \stackrel{\mathrm{D}}{\rightarrow}(2 \alpha+3) \sqrt{\frac{B(1+\alpha)}{2+\alpha}} \eta .
$$

Since $\eta$ can be written as $\eta=(1+\alpha) \int_{0}^{1} t^{\alpha}\left(W(1)-W\left(t^{2+\alpha}\right)\right) \mathrm{d} t$, we have

$$
\mathrm{E} \eta^{2}=\int_{0}^{1} \int_{0}^{1} s^{\alpha} t^{\alpha} R(t, s) \mathrm{d} s \mathrm{~d} t
$$

where

$$
R(t, s)=(1+\alpha)^{2} \mathrm{E}\left[\left(W(1)-W\left(s^{2+\alpha}\right)\right)\left(W(1)-W\left(t^{2+\alpha}\right)\right)\right] .
$$

We consider

$$
\mathrm{E} \eta^{2}=\int_{0}^{1} \int_{0}^{t} s^{\alpha} t^{\alpha} R(t, s) \mathrm{d} s \mathrm{~d} t+\int_{0}^{1} \int_{t}^{1} s^{\alpha} t^{\alpha} R(t, s) \mathrm{d} s \mathrm{~d} t .
$$

By a standard technique we find that the first term on the right-hand side of (29) is equal to

$$
\int_{0}^{1} \int_{0}^{t} s^{\alpha} t^{\alpha} \mathrm{E}\left[\left(W(1)-W\left(t^{2+\alpha}\right)\right)^{2}\right] \mathrm{d} s \mathrm{~d} t=\frac{\alpha+2}{2(3 \alpha+4)} .
$$

In a similar way we find that the second term on the right-hand side of (29) is also equal to $(\alpha+2) / 2(3 \alpha+4)$. Hence, we have

$$
\mathrm{E} \eta^{2}=\frac{\alpha+2}{3 \alpha+4}
$$

which implies the desired result. This completes the proof.

\section{Acknowledgements}

This paper is based on part of the results obtained under research project MS/THEOREM/ 335 funded by King Fahd University of Petroleum \& Minerals, Dhahran, Saudi Arabia. My sincere thanks to King Fahd University of Petroleum \& Minerals for all the support and facilities they provided. I am grateful to an anonymous referee for bringing to my attention Lemma 1, for carefully reading the first version of the paper, and for his/her valuable comments.

\section{References}

[1] Aliev, S. A. (1985). A limit theorem for Galton-Watson branching processes with immigration. Ukrain. Mat. Zh. 37, 656-659.

[2] Alzaid, A. A. And Al-Osh, M. (1990). An integer-valued $p$ th order autoregressive (INAR( $p$ )) process. J. Appl. Prob. 27, 314-324. 
[3] Bingham, N. H., Goldie, C. M. and Teugels, J. L. (1987). Regular Variation (Encyclopaedia Math. Appl. 27). Cambridge University Press.

[4] Dion, J. P., Gauthier, G. And Latour, A. (1995). Branching processes with immigration and integer-valued time series. Serdica Math. J. 21, 123-136.

[5] Feller, W. (1951). Diffusion processes in genetics. In Proc. Second Berkeley Symp. Math. Statist. Prob., ed. P. Neyman, University of California Press, Berkeley, CA, pp. 227-246.

[6] Franke, J. and Seligmann, T. (1993). Conditional maximum likelihood estimates for INAR(1) processes and their application to modelling epileptic seizure counts. In Developments in Time Series Analysis, ed. T. S. Rao, Chapman and Hall, London, pp. 310-330.

[7] Ispàny, M., Pap, G. and Van ZuiJlen, M. C. A. (2003). Asymptotic inference for nearly unstable INAR(1) models. J. Appl. Prob. 40, 750-765.

[8] Ispàny, M., Pap, G. And VAn Zuijlen, M. C. A. (2005). Fluctuation limit of branching processes with immigration and estimation of the means. Adv. Appl. Prob. 37, 523-538.

[9] Ispàny, M., PAP, G. ANd VAN ZuiJlen, M. C. A. (2006). Critical branching mechanisms with immigration and Ornstein-Uhlenbeck type diffusions. Acta Sci. Math. (Szeged) 71, 821-850.

[10] Jacod, J. And Shiryaev, A. N. (2003). Limit Theorems for Stochastic Processes. Springer, Berlin.

[11] Kawazu, K. and Watanabe, S. (1971). Branching processes with immigration and related limit theorems. Theory Prob. Appl. 16, 36-54.

[12] Lamperti, J. (1967a). Limiting distributions for branching processes. In Proc. Fifth Berkeley Symp. Math. Statist. Prob. University of California Press, Berkeley, CA, pp. 225-241.

[13] LAMPERTI, J. (1967b). The limit of a sequence of branching processes. Z. Wahrscheinlichkeitsth. 7, $271-288$.

[14] Lindvall, T. (1972). Convergence of critical Galton-Watson branching processes. J. Appl. Prob. 9, 445-450.

[15] Lindvall, T. (1974). Limit theorems for functionals of certain Galton-Watson branching processes. Adv. Appl. Prob. 6, 309-327.

[16] Rahimov, I. (1995). Random Sums and Branching Stochastic Processes (Lecture Notes Statist. 96). Springer, New York.

[17] SRIRAM, T. N. (1994). Invalidity of bootstrap for critical branching processes with immigration. Ann. Statist. 22, 1013-1023.

[18] WeI, C. Z. AND WINNICKI, J. (1989). Some asymptotic results for branching processes with immigration. Stoch. Process. Appl. 31, 261-282. 